\title{
Addition and Correction to:
}

Supporting Information

\section{Inorganic halide double perovskites with optoelectronic properties modulated by sublattice mixing}

Christopher J. Bartel, ${ }^{1 * \#}$ Jacob M. Clary, ${ }^{1}$ Christopher Sutton, ${ }^{2}$ Derek Vigil-Fowler, ${ }^{3}$ Bryan R. Goldsmith, ${ }^{4}$ Aaron M. Holder, ${ }^{1,3}$ Charles B. Musgrave ${ }^{*, 1,3,5,6,7}$

${ }^{1}$ Department of Chemical and Biological Engineering, University of Colorado Boulder, Boulder, CO 80309, USA

${ }^{2}$ Fritz-Haber-Institut der Max-Planck-Gesellschaft, Faradayweg 4-6, D-14195 Berlin, Germany

${ }^{3}$ Materials and Chemical Science and Technology Center, National Renewable Energy Laboratory, Golden, CO 80401, USA

${ }^{4}$ Department of Chemical Engineering, University of Michigan, Ann Arbor, MI 48109, USA

${ }^{5}$ Department of Chemistry, University of Colorado Boulder, Boulder, CO 80309, USA

${ }^{6}$ Renewable and Sustainable Energy Institute, University of Colorado Boulder, Boulder, CO 80309, USA

${ }^{7}$ Materials Science and Engineering Program, University of Colorado Boulder, Boulder, CO 80309, USA

\#present address: Department of Materials Science and Engineering, University of California, Berkeley, Berkeley, CA 94720, USA *correspondence to: christopher.bartel@colorado.edu, charles.musgrave@colorado.edu

Table S1. List of all $\mathrm{Cs}_{2} B B^{\prime} \mathrm{Cl}_{6}$ compounds screened in this study sorted by their $B$ and $B^{\prime}$ cations. For band gaps where functional is not explicitly listed, spHSE06@SCAN was used ("HSE" in the table header). $\Delta H_{\mathrm{d}}$ and $\Delta H_{\mathrm{f}}$ were calculated with SCAN. $\Delta H_{\mathrm{d}}$ values are color-coded to match the coloring in Figure 3. The last four columns prior to the final column provide whether each compound does (X) or does not (empty) meet certain screening criteria listed in the manuscript. The final column indicates whether the compound appears is newly included in this Addition.

\begin{tabular}{|c|c|c|c|c|c|c|c|c|c|c|c|c|}
\hline B & B' & $\begin{array}{c}\Delta H_{\mathrm{f}} \\
(\mathrm{eV} / \text { atom) }\end{array}$ & $\begin{array}{c}\Delta H_{\mathrm{d}} \\
(\mathrm{eV} / \text { atom) }\end{array}$ & $\begin{array}{c}E_{\mathrm{g}, \mathrm{PBE}} \\
(\mathrm{eV})\end{array}$ & $\begin{array}{c}E_{\mathrm{g}, \mathrm{SCAN}} \\
(\mathrm{eV})\end{array}$ & $\begin{array}{c}E_{\mathrm{g}, \mathrm{HSE}} \\
(\mathrm{eV})\end{array}$ & $\begin{array}{c}E_{\mathrm{g}, \mathrm{d}}-E_{\mathrm{g}} \\
(\mathrm{eV})\end{array}$ & $\begin{array}{c}\Delta H_{\mathrm{d}} \leq 0.05 \mathrm{eV} / \text { atom } \\
\text { (stable or nearly) }\end{array}$ & $\begin{array}{c}1 \leq E_{\mathrm{g}} \leq 3 \mathrm{eV} \\
\text { (semiconductor) }\end{array}$ & $\begin{array}{c}E_{\mathrm{g}, \mathrm{d}}-E_{\mathrm{g}} \leq 0.1 \mathrm{eV} \\
\text { (direct or nearly) }\end{array}$ & $\begin{array}{l}\text { non- } \\
\text { toxic }\end{array}$ & addition \\
\hline $\mathrm{Ag}$ & $\mathrm{Al}$ & -1.852 & 0.007 & 2.195 & 2.831 & 3.747 & 0 & $\mathrm{X}$ & & $\mathrm{X}$ & $\mathrm{X}$ & $\mathrm{X}$ \\
\hline $\mathrm{Ag}$ & As & -1.442 & -0.054 & 1.471 & 1.686 & 2.361 & 0.771 & $\mathrm{X}$ & $X$ & & & \\
\hline $\mathrm{Ag}$ & $\mathrm{Au}$ & -1.332 & 0.007 & 0.876 & 1.023 & 1.558 & 0.001 & $\mathrm{X}$ & $\mathrm{X}$ & $\mathrm{X}$ & $\mathrm{X}$ & \\
\hline $\mathrm{Ag}$ & $\mathrm{Bi}$ & -1.547 & -0.039 & 1.928 & 2.178 & 2.938 & 0.635 & $X$ & $X$ & & $X$ & \\
\hline $\mathrm{Ag}$ & Co & -1.441 & 0.025 & 0 & 0 & 0.321 & 0.417 & $\mathrm{X}$ & & & X & \\
\hline $\mathrm{Ag}$ & $\mathrm{Cr}$ & -1.781 & -0.097 & 0.98 & 1.986 & 2.7 & 0.393 & $\mathrm{X}$ & $\mathrm{X}$ & & X & X \\
\hline $\mathrm{Ag}$ & $\mathrm{Cu}$ & -1.374 & 0.041 & 0.305 & 0.329 & 0.578 & 0 & $\mathrm{X}$ & & $\mathrm{X}$ & $\mathrm{X}$ & \\
\hline $\mathrm{Ag}$ & $\mathrm{Fe}$ & -1.551 & -0.023 & 0.055 & 0.572 & 1.726 & 1.052 & $\mathrm{X}$ & $\mathrm{X}$ & & $\mathrm{X}$ & $\mathrm{X}$ \\
\hline $\mathrm{Ag}$ & $\mathrm{Ga}$ & -1.611 & 0.048 & 0.991 & 1.373 & 2.323 & 0 & X & X & $\mathrm{X}$ & X & \\
\hline $\mathrm{Ag}$ & In & -1.667 & -0.011 & 1.013 & 1.414 & 2.338 & 0 & $\mathrm{X}$ & $\mathrm{X}$ & $\mathrm{X}$ & $\mathrm{X}$ & \\
\hline $\mathrm{Ag}$ & Ir & -1.372 & -0.032 & 0.487 & 1.091 & 2.19 & 0 & X & X & X & $X$ & $X$ \\
\hline $\mathrm{Ag}$ & $\mathrm{Mn}$ & -1.525 & 0.125 & 0.628 & 1.244 & 2.683 & 0 & & $\mathrm{X}$ & $\mathrm{X}$ & $\mathrm{X}$ & \\
\hline $\mathrm{Ag}$ & Mo & -1.608 & -0.02 & 0.809 & 1.642 & 2.634 & 0 & $\mathrm{X}$ & $\mathrm{X}$ & $\mathrm{X}$ & $\mathrm{X}$ & $\mathrm{X}$ \\
\hline $\mathrm{Ag}$ & $\mathrm{Nb}$ & -1.715 & -0.061 & 0 & 0.473 & 1.61 & 0.005 & $X$ & $\mathrm{X}$ & $X$ & $\mathrm{X}$ & $X$ \\
\hline $\mathrm{Ag}$ & $\mathrm{Ni}$ & -1.456 & 0.06 & 0.05 & 0 & 0.431 & 0.355 & & & & X & \\
\hline $\mathrm{Ag}$ & $\mathrm{Pd}$ & -1.376 & -0.012 & 0 & 0.133 & 0.348 & 0.408 & $\mathrm{X}$ & & & $X$ & \\
\hline $\mathrm{Ag}$ & $\mathrm{Rh}$ & -1.482 & -0.075 & 0.499 & 1.185 & 2.29 & 0 & $\mathrm{X}$ & $\mathrm{X}$ & $\mathrm{X}$ & $\mathrm{X}$ & $\mathrm{X}$ \\
\hline
\end{tabular}




\begin{tabular}{|c|c|c|c|c|c|c|c|c|c|c|c|c|}
\hline $\mathrm{Ag}$ & $\mathrm{Sb}$ & -1.505 & 0.025 & 1.538 & 1.759 & 2.415 & 0.635 & $\mathrm{X}$ & $\mathrm{X}$ & & $\mathrm{X}$ & \\
\hline $\mathrm{Ag}$ & $\mathrm{Sc}$ & -2.093 & 0.02 & 3.23 & 3.584 & 4.834 & 0.037 & $\mathrm{X}$ & & $\mathrm{X}$ & $X$ & \\
\hline $\mathrm{Ag}$ & $\mathrm{Ta}$ & -1.568 & 0.215 & 0 & 0.135 & 1.283 & 0.001 & & $\mathrm{X}$ & $\mathrm{X}$ & $\mathrm{X}$ & \\
\hline $\mathrm{Ag}$ & $\mathrm{Ti}$ & -1.86 & 0.05 & 0 & 0.661 & 2.598 & 0 & & $\mathrm{X}$ & $\mathrm{X}$ & $\mathrm{X}$ & \\
\hline $\mathrm{Ag}$ & $\mathrm{Tl}$ & -1.451 & 0.013 & 0 & 0 & 0.265 & 0 & $\mathrm{X}$ & & $\mathrm{X}$ & & \\
\hline $\mathrm{Ag}$ & V & -1.747 & 0.035 & 0.23 & 1.102 & 2.98 & 0.018 & $\mathrm{X}$ & $\mathrm{X}$ & $\mathrm{X}$ & $\mathrm{X}$ & \\
\hline $\mathrm{Ag}$ & $\mathrm{Y}$ & -2.171 & -0.066 & 3.851 & 4.411 & 5.455 & 0.399 & $\mathrm{X}$ & & & $\mathrm{X}$ & \\
\hline $\mathrm{Al}$ & $\mathrm{Au}$ & -1.761 & 0.047 & 3.826 & 4.291 & 5.196 & 0.007 & $\mathrm{X}$ & & $\mathrm{X}$ & $\mathrm{X}$ & \\
\hline $\mathrm{Al}$ & $\mathrm{Hg}$ & -1.784 & 0.072 & 3.1 & 3.474 & 4.035 & 0.032 & & & $X$ & & \\
\hline $\mathrm{Al}$ & $\mathrm{K}$ & -2.139 & 0.007 & 4.76 & 5.482 & 6.158 & 0 & $\mathrm{X}$ & & $\mathrm{X}$ & $\mathrm{X}$ & \\
\hline $\mathrm{Al}$ & $\mathrm{Na}$ & -2.129 & 0.004 & 4.154 & 4.869 & 5.542 & 0 & $\mathrm{X}$ & & $\mathrm{X}$ & $\mathrm{X}$ & \\
\hline $\mathrm{Al}$ & $\mathrm{Rb}$ & -2.128 & 0.018 & 4.786 & 5.497 & 6.168 & 0 & $\mathrm{X}$ & & $\mathrm{X}$ & $\mathrm{X}$ & \\
\hline $\mathrm{Al}$ & $\mathrm{Tl}$ & -1.919 & 0.017 & 3.927 & 4.426 & 4.892 & 0.317 & $\mathrm{X}$ & & & & \\
\hline As & $\mathrm{Au}$ & -1.306 & 0.03 & 1.814 & 1.93 & 2.65 & 0.299 & $\mathrm{X}$ & $\mathrm{X}$ & & & \\
\hline As & $\mathrm{Hg}$ & -1.371 & 0.046 & 0.152 & 0.171 & 0.191 & 0.038 & $\mathrm{X}$ & & $X$ & & \\
\hline As & $\mathrm{K}$ & -1.727 & -0.053 & 3.598 & 3.857 & 4.489 & 0.009 & $\mathrm{X}$ & & $\mathrm{X}$ & & \\
\hline As & $\mathrm{Na}$ & -1.729 & -0.068 & 3.204 & 3.47 & 4.067 & 0.142 & $\mathrm{X}$ & & & & \\
\hline As & $\mathrm{Rb}$ & -1.72 & -0.045 & 3.648 & 3.909 & 4.543 & 0.016 & $\mathrm{X}$ & & $\mathrm{X}$ & & \\
\hline As & $\mathrm{Tl}$ & -1.525 & -0.061 & 1.576 & 1.786 & 2.183 & 0 & $\mathrm{X}$ & $\mathrm{X}$ & $\mathrm{X}$ & & \\
\hline $\mathrm{Au}$ & B & -1.45 & -0.001 & 4.13 & 4.464 & 5.435 & 0.127 & $\mathrm{X}$ & & & $\mathrm{X}$ & \\
\hline $\mathrm{Au}$ & $\mathrm{Bi}$ & -1.415 & 0.056 & 2.272 & 2.396 & 3.207 & 0.283 & & & & $\mathrm{X}$ & \\
\hline $\mathrm{Au}$ & Co & -1.361 & 0.072 & 0.272 & 0.495 & 0.593 & 0.002 & & & $\mathrm{X}$ & $\mathrm{X}$ & \\
\hline $\mathrm{Au}$ & $\mathrm{Cr}$ & -1.611 & 0.021 & 1.305 & 2.297 & 3.184 & 0 & $\mathrm{X}$ & & $\mathrm{X}$ & $\mathrm{X}$ & \\
\hline $\mathrm{Au}$ & $\mathrm{Cu}$ & -1.352 & 0.03 & 0.754 & 0.81 & 1.298 & 0 & $\mathrm{X}$ & $\mathrm{X}$ & $\mathrm{X}$ & $\mathrm{X}$ & \\
\hline $\mathrm{Au}$ & $\mathrm{Fe}$ & -1.48 & 0.013 & 0.911 & 1.385 & 2.712 & 0.013 & $\mathrm{X}$ & $\mathrm{X}$ & $\mathrm{X}$ & $\mathrm{X}$ & \\
\hline $\mathrm{Au}$ & $\mathrm{Ga}$ & -1.505 & 0.102 & 2.592 & 2.856 & 3.736 & 0.013 & & & $\mathrm{X}$ & $\mathrm{X}$ & \\
\hline $\mathrm{Au}$ & $\mathrm{Hg}$ & -1.306 & 0.068 & 0 & 0.409 & 1.113 & 0.341 & & $\mathrm{X}$ & & & \\
\hline $\mathrm{Au}$ & In & -1.547 & 0.074 & 2.392 & 2.655 & 3.504 & 0 & & & $\mathrm{X}$ & $\mathrm{X}$ & \\
\hline $\mathrm{Au}$ & $\mathrm{Ir}$ & -1.263 & 0.041 & 1.556 & 2.058 & 3.214 & 0.086 & $\mathrm{X}$ & & $\mathrm{X}$ & $\mathrm{X}$ & $\mathrm{X}$ \\
\hline $\mathrm{Au}$ & $\mathrm{K}$ & -1.553 & 0.016 & 1.662 & 1.945 & 2.706 & 0.076 & $\mathrm{X}$ & $\mathrm{X}$ & $\mathrm{X}$ & $\mathrm{X}$ & \\
\hline $\mathrm{Au}$ & $\mathrm{Li}$ & -1.557 & -0.019 & 1.512 & 1.806 & 2.606 & 0.071 & $\mathrm{X}$ & $\mathrm{X}$ & $X$ & $\mathrm{X}$ & \\
\hline $\mathrm{Au}$ & $\mathrm{Mn}$ & -1.566 & 0.05 & 0.884 & 0.792 & 1.141 & 0.168 & & $\mathrm{X}$ & & $\mathrm{X}$ & \\
\hline $\mathrm{Au}$ & Mo & -1.477 & 0.112 & 1.03 & 1.715 & 3.057 & 0.045 & & & $X$ & $\mathrm{X}$ & $\mathrm{X}$ \\
\hline $\mathrm{Au}$ & $\mathrm{Na}$ & -1.554 & -0.026 & 1.264 & 1.552 & 2.367 & 0.023 & $\mathrm{X}$ & $\mathrm{X}$ & $\mathrm{X}$ & $\mathrm{X}$ & \\
\hline $\mathrm{Au}$ & $\mathrm{Nb}$ & -1.599 & 0.04 & 0.173 & 0.707 & 1.816 & 0.045 & $\mathrm{X}$ & $X$ & $\mathrm{X}$ & $\mathrm{X}$ & X \\
\hline $\mathrm{Au}$ & $\mathrm{Ni}$ & -1.389 & 0.094 & 0.121 & 0.854 & 1.554 & 0.882 & & $\mathrm{X}$ & & $\mathrm{X}$ & \\
\hline $\mathrm{Au}$ & $\mathrm{Pd}$ & -1.301 & 0.03 & 0.042 & 0.091 & 0 & 0 & $\mathrm{X}$ & & $\mathrm{X}$ & $\mathrm{X}$ & \\
\hline $\mathrm{Au}$ & $\mathrm{Rb}$ & -1.578 & 0.013 & 1.643 & 1.928 & 2.701 & 0.098 & $X$ & $X$ & $\mathrm{X}$ & $\mathrm{X}$ & \\
\hline $\mathrm{Au}$ & $\mathrm{Rh}$ & -1.328 & 0.028 & 1.256 & 1.815 & 2.995 & 0.111 & $\mathrm{X}$ & $X$ & & $\mathrm{X}$ & \\
\hline $\mathrm{Au}$ & $\mathrm{Ru}$ & -1.243 & 0.162 & 0 & 0.423 & 1.66 & 0.178 & & $\mathrm{X}$ & & $\mathrm{X}$ & \\
\hline $\mathrm{Au}$ & $\mathrm{Sb}$ & -1.372 & 0.123 & 1.796 & 1.897 & 2.627 & 0.272 & & $X$ & & $\mathrm{X}$ & \\
\hline $\mathrm{Au}$ & $\mathrm{Sc}$ & -1.96 & 0.118 & 2.976 & 3.228 & 4.365 & 0.072 & & & X & $\mathrm{X}$ & \\
\hline
\end{tabular}




\begin{tabular}{|c|c|c|c|c|c|c|c|c|c|c|c|c|}
\hline $\mathrm{Au}$ & $\mathrm{Ta}$ & -1.496 & 0.288 & 0.539 & 0.712 & 0.855 & 0.114 & & & & $\mathrm{X}$ & \\
\hline $\mathrm{Au}$ & $\mathrm{Ti}$ & -1.742 & 0.168 & 0.186 & 0.742 & 2.766 & 0.025 & & $\mathrm{X}$ & $\mathrm{X}$ & $\mathrm{X}$ & \\
\hline $\mathrm{Au}$ & $\mathrm{Tl}$ & -1.358 & 0.073 & 0 & 0 & 0 & 0 & & & $\mathrm{X}$ & & \\
\hline $\mathrm{Au}$ & $\mathrm{V}$ & -1.636 & 0.111 & 0.194 & 1.166 & 2.879 & 0.009 & & $\mathrm{X}$ & $\mathrm{X}$ & $\mathrm{X}$ & \\
\hline $\mathrm{Au}$ & $\mathrm{Y}$ & -2.046 & 0.006 & 3.969 & 4.326 & 5.427 & 0 & $\mathrm{X}$ & & $\mathrm{X}$ & $\mathrm{X}$ & \\
\hline B & $\mathrm{Hg}$ & -1.489 & 0.026 & 2.745 & 2.53 & 3.222 & 0.076 & $\mathrm{X}$ & & $\mathrm{X}$ & & X \\
\hline B & $\mathrm{K}$ & -1.785 & 0.002 & 4.396 & 4.978 & 5.633 & 0 & $\mathrm{X}$ & & $\mathrm{X}$ & $\mathrm{X}$ & \\
\hline B & $\mathrm{Rb}$ & -1.813 & -0.026 & 4.724 & 5.275 & 5.997 & 0 & $\mathrm{X}$ & & $\mathrm{X}$ & $\mathrm{X}$ & \\
\hline B & $\mathrm{Tl}$ & -1.594 & -0.017 & 3.847 & 4.117 & 4.834 & 0.11 & $\mathrm{X}$ & & & & \\
\hline $\mathrm{Ba}$ & $\mathrm{Be}$ & -2.256 & 0.011 & 4.77 & 5.244 & 6.004 & 0 & $X$ & & X & $\mathrm{X}$ & \\
\hline $\mathrm{Ba}$ & $\mathrm{Ca}$ & -2.601 & 0.074 & 5.407 & 6 & 6.713 & 0.034 & & & $\mathrm{X}$ & $X$ & \\
\hline $\mathrm{Ba}$ & $\mathrm{Cd}$ & -2.173 & 0.053 & 4.394 & 5.012 & 5.794 & 0.109 & & & & & \\
\hline $\mathrm{Ba}$ & Co & -2.067 & 0.034 & 0.64 & 1.955 & 4.538 & 0.125 & $\mathrm{X}$ & & & $\mathrm{X}$ & \\
\hline $\mathrm{Ba}$ & $\mathrm{Cr}$ & -2.199 & 0.061 & 0.507 & 1.266 & 3.258 & 0.071 & & & $\mathrm{X}$ & $\mathrm{X}$ & \\
\hline $\mathrm{Ba}$ & $\mathrm{Cu}$ & -1.974 & 0.105 & 0.587 & 1.313 & 2.955 & 0.08 & & $\mathrm{X}$ & $\mathrm{X}$ & $\mathrm{X}$ & \\
\hline $\mathrm{Ba}$ & $\mathrm{Ge}$ & -2.034 & 0.074 & 3.959 & 4.298 & 4.935 & 0.02 & & & $\mathrm{X}$ & $\mathrm{X}$ & \\
\hline $\mathrm{Ba}$ & $\mathrm{Hg}$ & -1.971 & 0.095 & 2.978 & 3.353 & 4.296 & 0.313 & & & & & \\
\hline $\mathrm{Ba}$ & $\mathrm{Mg}$ & -2.423 & 0.037 & 5.16 & 5.816 & 6.483 & 0 & $X$ & & X & $X$ & \\
\hline $\mathrm{Ba}$ & $\mathrm{Mn}$ & -2.253 & 0.071 & 2.448 & 3.562 & 5.415 & 0.355 & & & & $\mathrm{X}$ & \\
\hline $\mathrm{Ba}$ & $\mathrm{Ni}$ & -2.008 & 0.143 & 0.729 & 1.632 & 3.694 & 0.102 & & & & $\mathrm{X}$ & \\
\hline $\mathrm{Ba}$ & $\mathrm{Pb}$ & -2.201 & 0.004 & 4.484 & 4.85 & 5.506 & 0.033 & $\mathrm{X}$ & & $\mathrm{X}$ & & X \\
\hline $\mathrm{Ba}$ & $\mathrm{Pd}$ & -1.968 & 0.06 & 1.995 & 2.371 & 3.639 & 0.043 & & & $\mathrm{X}$ & $\mathrm{X}$ & \\
\hline $\mathrm{Ba}$ & $\mathrm{Pt}$ & -1.904 & 0.107 & 2.302 & 2.766 & 3.904 & 0.022 & & & $\mathrm{X}$ & $\mathrm{X}$ & \\
\hline $\mathrm{Ba}$ & $\mathrm{Sn}$ & -2.096 & 0.05 & 3.753 & 4.096 & 4.673 & 0.002 & & & $\mathrm{X}$ & $\mathrm{X}$ & \\
\hline $\mathrm{Ba}$ & $\mathrm{Sr}$ & -2.644 & -0.013 & 5.111 & 5.708 & 6.466 & 0 & $\mathrm{X}$ & & $\mathrm{X}$ & $\mathrm{X}$ & $\mathrm{X}$ \\
\hline $\mathrm{Ba}$ & $\mathrm{Ti}$ & -2.302 & 0.037 & 0.257 & 1.008 & 2.714 & 0.042 & $\mathrm{X}$ & $\mathrm{X}$ & $\mathrm{X}$ & $\mathrm{X}$ & \\
\hline $\mathrm{Ba}$ & $\mathrm{V}$ & -2.288 & 0.053 & 1.474 & 2.618 & 4.217 & 0 & & & $\mathrm{X}$ & $\mathrm{X}$ & \\
\hline $\mathrm{Ba}$ & $\mathrm{Zn}$ & -2.194 & 0.095 & 4.272 & 5.151 & 5.994 & 0.004 & & & $\mathrm{X}$ & $\mathrm{X}$ & $\mathrm{X}$ \\
\hline $\mathrm{Be}$ & $\mathrm{Ca}$ & -2.281 & 0.019 & 5.05 & 5.742 & 6.471 & 0 & $\mathrm{X}$ & & $\mathrm{X}$ & $X$ & $\mathrm{X}$ \\
\hline $\mathrm{Be}$ & $\mathrm{Hg}$ & -1.674 & 0.016 & 3.096 & 3.508 & 4.553 & 0.003 & $\mathrm{X}$ & & $\mathrm{X}$ & & X \\
\hline $\mathrm{Be}$ & $\mathrm{Pb}$ & -1.831 & -0.001 & 4.07 & 4.405 & 5.091 & 0.085 & $\mathrm{X}$ & & $\mathrm{X}$ & & \\
\hline $\mathrm{Be}$ & $\mathrm{Sn}$ & -1.784 & -0.013 & 3.907 & 4.24 & 4.843 & 0.064 & $\mathrm{X}$ & & $\mathrm{X}$ & $\mathrm{X}$ & \\
\hline $\mathrm{Be}$ & $\mathrm{Sr}$ & -2.253 & 0.004 & 4.942 & 5.474 & 6.206 & 0 & $\mathrm{X}$ & & $\mathrm{X}$ & $\mathrm{X}$ & \\
\hline $\mathrm{Bi}$ & $\mathrm{Cu}$ & -1.516 & -0.007 & 1.782 & 1.892 & 3.047 & 0.174 & $\mathrm{X}$ & & & $\mathrm{X}$ & \\
\hline $\mathrm{Bi}$ & $\mathrm{Hg}$ & -1.452 & 0.066 & 0.111 & 0.127 & 0.138 & 0.024 & & & $\mathrm{X}$ & & \\
\hline $\mathrm{Bi}$ & $\mathrm{K}$ & -1.846 & 0.006 & 4.177 & 4.489 & 5.131 & 0 & $\mathrm{X}$ & & $\mathrm{X}$ & $\mathrm{X}$ & \\
\hline $\mathrm{Bi}$ & $\mathrm{Li}$ & -1.828 & -0.025 & 3.652 & 3.973 & 4.564 & 0.094 & $X$ & & $\mathrm{X}$ & $X$ & \\
\hline $\mathrm{Bi}$ & $\mathrm{Na}$ & -1.844 & -0.03 & 3.846 & 4.167 & 4.78 & 0.129 & $\mathrm{X}$ & & & $\mathrm{X}$ & \\
\hline $\mathrm{Bi}$ & $\mathrm{Pd}$ & -1.439 & 0.048 & 0 & 0 & 0.068 & 0 & $\mathrm{X}$ & & $\mathrm{X}$ & $\mathrm{X}$ & \\
\hline $\mathrm{Bi}$ & $\mathrm{Rb}$ & -1.877 & -0.049 & 4.177 & 4.457 & 5.151 & 0 & $\mathrm{X}$ & & $\mathrm{X}$ & $\mathrm{X}$ & X \\
\hline $\mathrm{Bi}$ & $\mathrm{Tl}$ & -1.669 & -0.051 & 2.572 & 2.602 & 3.097 & 0 & $\mathrm{X}$ & & $\mathrm{X}$ & & X \\
\hline $\mathrm{Ca}$ & $\mathrm{Cd}$ & -2.192 & 0.024 & 4.058 & 4.676 & 5.546 & 0 & $\mathrm{X}$ & & $\mathrm{X}$ & & \\
\hline
\end{tabular}




\begin{tabular}{|c|c|c|c|c|c|c|c|c|c|c|c|c|}
\hline $\mathrm{Ca}$ & Co & -2.085 & 0.026 & 0.611 & 1.93 & 4.526 & 0.049 & $\mathrm{X}$ & & $\mathrm{X}$ & $\mathrm{X}$ & \\
\hline $\mathrm{Ca}$ & $\mathrm{Cr}$ & -2.215 & 0.036 & 0.358 & 1.117 & 3.169 & 0.009 & $\mathrm{X}$ & & $\mathrm{X}$ & $\mathrm{X}$ & \\
\hline $\mathrm{Ca}$ & $\mathrm{Cu}$ & -1.984 & 0.056 & 0.505 & 1.119 & 2.73 & 0.092 & & $\mathrm{X}$ & $\mathrm{X}$ & $X$ & \\
\hline $\mathrm{Ca}$ & $\mathrm{Fe}$ & -2.115 & 0.01 & 0.072 & 0.91 & 3.69 & 0.024 & $\mathrm{X}$ & & $\mathrm{X}$ & $\mathrm{X}$ & \\
\hline $\mathrm{Ca}$ & $\mathrm{Ge}$ & -2.052 & 0.046 & 3.943 & 4.294 & 4.924 & 0 & $X$ & & $\mathrm{X}$ & $X$ & \\
\hline $\mathrm{Ca}$ & $\mathrm{Hg}$ & -1.984 & 0.047 & 2.706 & 3.089 & 4.045 & 0.198 & $X$ & & & & \\
\hline $\mathrm{Ca}$ & $\mathrm{Mg}$ & -2.444 & 0.005 & 5.396 & 6.162 & 6.788 & 0 & $\mathrm{X}$ & & $\mathrm{X}$ & $\mathrm{X}$ & \\
\hline $\mathrm{Ca}$ & $\mathrm{Mn}$ & -2.276 & 0.002 & 2.478 & 3.614 & 5.573 & 0.563 & X & & & $\mathrm{X}$ & \\
\hline $\mathrm{Ca}$ & $\mathrm{Ni}$ & -2.02 & 0.124 & 0.779 & 1.678 & 3.672 & 0.123 & & & & $\mathrm{X}$ & \\
\hline $\mathrm{Ca}$ & $\mathrm{Pb}$ & -2.173 & 0.03 & 4.46 & 4.858 & 5.496 & 0.02 & $\mathrm{X}$ & & $X$ & & \\
\hline $\mathrm{Ca}$ & $\mathrm{Pd}$ & -1.939 & 0.063 & 1.364 & 1.711 & 3.078 & 0.071 & & & $\mathrm{X}$ & $\mathrm{X}$ & \\
\hline $\mathrm{Ca}$ & $\mathrm{Pt}$ & -1.913 & 0.088 & 2.383 & 2.861 & 3.989 & 0 & & & $\mathrm{X}$ & $\mathrm{X}$ & \\
\hline $\mathrm{Ca}$ & $\mathrm{Sn}$ & -2.111 & 0.033 & 3.779 & 4.145 & 4.71 & 0.006 & $\mathrm{X}$ & & $\mathrm{X}$ & $\mathrm{X}$ & \\
\hline $\mathrm{Ca}$ & $\mathrm{Sr}$ & -2.619 & 0.036 & 5.446 & 6.09 & 6.768 & 0.014 & X & & $\mathrm{X}$ & $\mathrm{X}$ & \\
\hline $\mathrm{Ca}$ & $\mathrm{Ti}$ & -2.322 & 0.006 & 0.224 & 0.987 & 2.732 & 0.072 & $\mathrm{X}$ & $X$ & $\mathrm{X}$ & $\mathrm{X}$ & \\
\hline $\mathrm{Ca}$ & V & -2.307 & 0.024 & 1.332 & 2.489 & 4.214 & 0 & $\mathrm{X}$ & & $\mathrm{X}$ & $\mathrm{X}$ & \\
\hline $\mathrm{Ca}$ & $\mathrm{Zn}$ & -2.171 & 0.066 & 4.043 & 4.662 & 5.572 & 0 & & & $\mathrm{X}$ & $\mathrm{X}$ & \\
\hline $\mathrm{Cd}$ & $\mathrm{Co}$ & -1.653 & 0.033 & 0.576 & 1.852 & 3.389 & 0 & $X$ & & $\mathrm{X}$ & & \\
\hline $\mathrm{Cd}$ & $\mathrm{Cr}$ & -1.796 & 0.049 & 0.256 & 0.681 & 2.222 & 0 & $\mathrm{X}$ & $X$ & $\mathrm{X}$ & & \\
\hline $\mathrm{Cd}$ & $\mathrm{Cu}$ & -1.574 & 0.06 & 0.215 & 0.821 & 2.177 & 0.295 & & $\mathrm{X}$ & & & \\
\hline $\mathrm{Cd}$ & $\mathrm{Fe}$ & -1.687 & 0.032 & 0 & 0.604 & 2.843 & 0.325 & $\mathrm{X}$ & $\mathrm{X}$ & & & \\
\hline $\mathrm{Cd}$ & $\mathrm{Ge}$ & -1.64 & 0.053 & 2.116 & 2.453 & 3.044 & 0.592 & & & & & \\
\hline $\mathrm{Cd}$ & $\mathrm{Hg}$ & -1.567 & 0.059 & 0.924 & 1.24 & 2.02 & 0.097 & & $\mathrm{X}$ & $\mathrm{X}$ & & \\
\hline $\mathrm{Cd}$ & $\mathrm{Mg}$ & -2.009 & 0.034 & 2.859 & 3.506 & 4.307 & 0 & $\mathrm{X}$ & & $\mathrm{X}$ & & \\
\hline $\mathrm{Cd}$ & $\mathrm{Mn}$ & -1.849 & 0.02 & 1.21 & 1.896 & 3.038 & 0 & $\mathrm{X}$ & & $\mathrm{X}$ & & \\
\hline $\mathrm{Cd}$ & $\mathrm{Ni}$ & -1.593 & 0.141 & 0.489 & 1.358 & 3.167 & 0.364 & & & & & \\
\hline $\mathrm{Cd}$ & $\mathrm{Pb}$ & -1.756 & 0.033 & 2.818 & 3.238 & 3.839 & 0.545 & $X$ & & & & \\
\hline $\mathrm{Cd}$ & $\mathrm{Pd}$ & -1.539 & 0.045 & 1.046 & 1.309 & 2.49 & 0.333 & $\mathrm{X}$ & $X$ & & & \\
\hline $\mathrm{Cd}$ & $\mathrm{Pt}$ & -1.486 & 0.11 & 1.824 & 2.241 & 3.336 & 0.027 & & & $X$ & & \\
\hline $\mathrm{Cd}$ & $\mathrm{Sn}$ & -1.699 & 0.032 & 2.066 & 2.428 & 2.96 & 0.565 & $\mathrm{X}$ & $\mathrm{X}$ & & & \\
\hline $\mathrm{Cd}$ & $\mathrm{Sr}$ & -2.194 & 0.022 & 4.236 & 4.887 & 5.712 & 0.003 & $\mathrm{X}$ & & $\mathrm{X}$ & & \\
\hline $\mathrm{Cd}$ & $\mathrm{Ti}$ & -1.887 & 0.092 & 0.075 & 0.76 & 2.005 & 0.004 & & $\mathrm{X}$ & $\mathrm{X}$ & & \\
\hline $\mathrm{Cd}$ & $\mathrm{V}$ & -1.868 & 0.057 & 0.192 & 1.2 & 2.79 & 0 & & $\mathrm{X}$ & $\mathrm{X}$ & & \\
\hline $\mathrm{Cd}$ & $\mathrm{Zn}$ & -1.739 & 0.076 & 3.629 & 4.14 & 5.027 & 0 & & & $\mathrm{X}$ & & \\
\hline Co & $\mathrm{Cr}$ & -1.686 & 0.034 & 0.143 & 0.115 & 2.243 & 0.074 & $\mathrm{X}$ & $\mathrm{X}$ & $\mathrm{X}$ & $\mathrm{X}$ & \\
\hline Co & $\mathrm{Fe}$ & -1.557 & 0.037 & 0.122 & 0.532 & 3.238 & 0 & $\mathrm{X}$ & & $\mathrm{X}$ & $\mathrm{X}$ & \\
\hline Co & $\mathrm{Ge}$ & -1.545 & 0.022 & 0.707 & 1.888 & 3.425 & 0.202 & $\mathrm{X}$ & & & $\mathrm{X}$ & \\
\hline Co & $\mathrm{Hg}$ & -1.463 & 0.038 & 0.315 & 1.168 & 2.478 & 0.131 & $\mathrm{X}$ & $\mathrm{X}$ & & & \\
\hline Co & $\mathrm{K}$ & -1.76 & -0.092 & 1.157 & 2.32 & 3.346 & 0 & $\mathrm{X}$ & & $\mathrm{X}$ & $\mathrm{X}$ & \\
\hline Co & $\mathrm{Mg}$ & -1.926 & -0.008 & 0.538 & 1.799 & 4.219 & 0 & $\mathrm{X}$ & & $\mathrm{X}$ & $\mathrm{X}$ & $\mathrm{X}$ \\
\hline Co & $\mathrm{Mn}$ & -1.738 & 0.022 & 0.554 & 1.772 & 4.043 & 0.045 & $\mathrm{X}$ & & $\mathrm{X}$ & $X$ & \\
\hline Co & $\mathrm{Ni}$ & -1.596 & 0.014 & 0.242 & 1.516 & 2.721 & 0.232 & $\mathrm{X}$ & $\mathrm{X}$ & & $\mathrm{X}$ & $\mathrm{X}$ \\
\hline
\end{tabular}




\begin{tabular}{|c|c|c|c|c|c|c|c|c|c|c|c|c|}
\hline Co & $\mathrm{Pb}$ & -1.654 & 0.01 & 0.368 & 1.398 & 3.234 & 1.044 & $\mathrm{X}$ & & & & \\
\hline Co & $\mathrm{Pt}$ & -1.401 & 0.07 & 0.968 & 1.978 & 3.602 & 0.109 & & & & $\mathrm{X}$ & \\
\hline Co & $\mathrm{Rb}$ & -1.753 & -0.084 & 1.22 & 2.436 & 3.457 & 0 & $\mathrm{X}$ & & $\mathrm{X}$ & $X$ & \\
\hline Co & $\mathrm{Sn}$ & -1.597 & 0.009 & 0 & 0.915 & 2.589 & 0.998 & $\mathrm{X}$ & $\mathrm{X}$ & & $\mathrm{X}$ & \\
\hline Co & $\mathrm{Sr}$ & -2.091 & 0 & 0.58 & 1.918 & 4.509 & 0.042 & $X$ & & $\mathrm{X}$ & $\mathrm{X}$ & \\
\hline Co & $\mathrm{Ti}$ & -1.847 & 0.069 & 0 & 0.112 & 1.104 & 0.604 & & $\mathrm{X}$ & & $\mathrm{X}$ & $\mathrm{X}$ \\
\hline Co & $\mathrm{Tl}$ & -1.563 & -0.004 & 1.085 & 2.176 & 2.805 & 0.05 & $\mathrm{X}$ & $\mathrm{X}$ & $\mathrm{X}$ & & \\
\hline Co & V & -1.791 & 0.02 & 0 & 0 & 2.441 & 0.023 & X & $X$ & $\mathrm{X}$ & $\mathrm{X}$ & $\mathrm{X}$ \\
\hline Co & $\mathrm{Zn}$ & -1.644 & 0.081 & 0.914 & 2.07 & 4.461 & 0.011 & & & $\mathrm{X}$ & $\mathrm{X}$ & \\
\hline $\mathrm{Cr}$ & $\mathrm{Fe}$ & -1.721 & 0.033 & 0 & 0.758 & 3.056 & 0.33 & $\mathrm{X}$ & & & $\mathrm{X}$ & \\
\hline $\mathrm{Cr}$ & $\mathrm{Ge}$ & -1.702 & 0.025 & 0 & 0 & 1.151 & 0.201 & $\mathrm{X}$ & $\mathrm{X}$ & & $\mathrm{X}$ & $\mathrm{X}$ \\
\hline $\mathrm{Cr}$ & $\mathrm{Hg}$ & -1.652 & 0.042 & 0 & 0 & 0.34 & 0.848 & $\mathrm{X}$ & & & & \\
\hline $\mathrm{Cr}$ & $\mathrm{K}$ & -2.051 & -0.08 & 1.816 & 2.988 & 3.993 & 0.044 & $\mathrm{X}$ & & $\mathrm{X}$ & $\mathrm{X}$ & \\
\hline $\mathrm{Cr}$ & $\mathrm{Mg}$ & -2.029 & 0.049 & 0.428 & 1.147 & 3.112 & 0.146 & X & & & $\mathrm{X}$ & \\
\hline $\mathrm{Cr}$ & $\mathrm{Mn}$ & -1.873 & 0.03 & 0.285 & 0.79 & 2.472 & 0.05 & $\mathrm{X}$ & $X$ & $\mathrm{X}$ & $\mathrm{X}$ & \\
\hline $\mathrm{Cr}$ & $\mathrm{Na}$ & -2.043 & -0.085 & 1.538 & 2.687 & 3.56 & 0.045 & $\mathrm{X}$ & & $\mathrm{X}$ & X & \\
\hline $\mathrm{Cr}$ & $\mathrm{Ni}$ & -1.735 & 0.034 & 0 & 0 & 1.903 & 0.174 & $X$ & $\mathrm{X}$ & & $\mathrm{X}$ & $X$ \\
\hline $\mathrm{Cr}$ & $\mathrm{Pb}$ & -1.781 & 0.042 & 0 & 0.301 & 1.871 & 0.27 & $X$ & $\mathrm{X}$ & & & \\
\hline $\mathrm{Cr}$ & $\mathrm{Pt}$ & -1.538 & 0.113 & 0.526 & 0.988 & 2.634 & 0.106 & & $\mathrm{X}$ & & $\mathrm{X}$ & \\
\hline $\mathrm{Cr}$ & $\mathrm{Rb}$ & -2.083 & -0.112 & 1.854 & 3.062 & 4.103 & 0.043 & $\mathrm{X}$ & & $\mathrm{X}$ & $\mathrm{X}$ & X \\
\hline $\mathrm{Cr}$ & $\mathrm{Sn}$ & -1.763 & 0.002 & 0 & 0 & 1.209 & 0.203 & $\mathrm{X}$ & $\mathrm{X}$ & & $\mathrm{X}$ & $\mathrm{X}$ \\
\hline $\mathrm{Cr}$ & $\mathrm{Sr}$ & -2.218 & 0.032 & 0.384 & 1.148 & 3.191 & 0.038 & $\mathrm{X}$ & & $\mathrm{X}$ & $\mathrm{X}$ & \\
\hline $\mathrm{Cr}$ & $\mathrm{Ti}$ & -1.951 & 0.045 & 0 & 0.185 & 1.342 & 0 & $X$ & $\mathrm{X}$ & $\mathrm{X}$ & $\mathrm{X}$ & $\mathrm{X}$ \\
\hline $\mathrm{Cr}$ & $\mathrm{Tl}$ & -1.838 & -0.077 & 1.745 & 2.821 & 3.302 & 0.054 & $\mathrm{X}$ & & $\mathrm{X}$ & & \\
\hline $\mathrm{Cr}$ & $\mathrm{V}$ & -1.886 & 0.074 & 0 & 0.997 & 2.772 & 0.39 & & $\mathrm{X}$ & & $\mathrm{X}$ & \\
\hline $\mathrm{Cr}$ & $\mathrm{Zn}$ & -1.781 & 0.068 & 0.928 & 1.55 & 3.443 & 0.042 & & & $\mathrm{X}$ & $\mathrm{X}$ & \\
\hline $\mathrm{Cu}$ & $\mathrm{Ge}$ & -1.505 & 0.011 & 0 & 0 & 0 & 0 & $X$ & & $\mathrm{X}$ & $X$ & X \\
\hline $\mathrm{Cu}$ & $\mathrm{Hg}$ & -1.415 & 0.034 & 0.306 & 0.89 & 2.224 & 0.742 & $\mathrm{X}$ & $X$ & & & \\
\hline $\mathrm{Cu}$ & In & -1.633 & 0.026 & 0.87 & 1.037 & 2.191 & 0 & $X$ & $X$ & $\mathrm{X}$ & $X$ & \\
\hline $\mathrm{Cu}$ & $\mathrm{Ir}$ & -1.343 & 0.037 & 0.98 & 1.512 & 2.675 & 0.028 & $\mathrm{X}$ & $\mathrm{X}$ & $\mathrm{X}$ & $\mathrm{X}$ & $\mathrm{X}$ \\
\hline $\mathrm{Cu}$ & $\mathrm{K}$ & -1.554 & 0.091 & 0.831 & 0.964 & 1.617 & 0.082 & & $\mathrm{X}$ & $\mathrm{X}$ & $\mathrm{X}$ & \\
\hline $\mathrm{Cu}$ & $\mathrm{Mg}$ & -1.84 & 0.027 & 0.355 & 1.051 & 2.62 & 0.039 & $\mathrm{X}$ & $\mathrm{X}$ & $\mathrm{X}$ & $\mathrm{X}$ & X \\
\hline $\mathrm{Cu}$ & Mo & -1.573 & 0.017 & 1.398 & 2.001 & 3.121 & 0.027 & $X$ & & $\mathrm{X}$ & $\mathrm{X}$ & $\mathrm{X}$ \\
\hline $\mathrm{Cu}$ & $\mathrm{Na}$ & -1.571 & 0.061 & 0.461 & 0.547 & 1.206 & 0.157 & & $\mathrm{X}$ & & $\mathrm{X}$ & \\
\hline $\mathrm{Cu}$ & $\mathrm{Nb}$ & -1.679 & -0.019 & 0 & 0.464 & 1.57 & 0.003 & $\mathrm{X}$ & $\mathrm{X}$ & $\mathrm{X}$ & $\mathrm{X}$ & $\mathrm{X}$ \\
\hline $\mathrm{Cu}$ & $\mathrm{Pb}$ & -1.555 & 0.059 & 0 & 0.064 & 0.125 & 0.541 & & & & & \\
\hline $\mathrm{Cu}$ & $\mathrm{Pt}$ & -1.321 & 0.098 & 0 & 0.047 & 0.9 & 0.406 & & & & $X$ & \\
\hline $\mathrm{Cu}$ & $\mathrm{Rb}$ & -1.577 & 0.069 & 0.056 & 0.248 & 1.077 & 0.004 & & $\mathrm{X}$ & $\mathrm{X}$ & $\mathrm{X}$ & \\
\hline $\mathrm{Cu}$ & $\mathrm{Rh}$ & -1.457 & -0.013 & 0.764 & 0.905 & 1.947 & 0.082 & $\mathrm{X}$ & $\mathrm{X}$ & $\mathrm{X}$ & $\mathrm{X}$ & $\mathrm{X}$ \\
\hline $\mathrm{Cu}$ & $\mathrm{Ru}$ & -1.404 & 0.043 & 0 & 0.425 & 1.85 & 0.006 & $\mathrm{X}$ & $\mathrm{X}$ & $\mathrm{X}$ & $\mathrm{X}$ & $\mathrm{X}$ \\
\hline $\mathrm{Cu}$ & $\mathrm{Sb}$ & -1.48 & 0.053 & 1.65 & 1.768 & 2.809 & 0.245 & & $X$ & & $\mathrm{X}$ & \\
\hline $\mathrm{Cu}$ & $\mathrm{Sc}$ & -2.056 & 0.06 & 2.064 & 2.259 & 3.739 & 0.004 & & & $\mathrm{X}$ & $\mathrm{X}$ & \\
\hline
\end{tabular}




\begin{tabular}{|c|c|c|c|c|c|c|c|c|c|c|c|c|}
\hline $\mathrm{Cu}$ & $\mathrm{Sn}$ & -1.544 & 0.062 & 0 & 0 & 0.048 & 0.368 & & & & $\mathrm{X}$ & \\
\hline $\mathrm{Cu}$ & $\mathrm{Sr}$ & -1.99 & 0.07 & 0.618 & 1.247 & 2.973 & 0.014 & & $\mathrm{X}$ & $\mathrm{X}$ & $\mathrm{X}$ & \\
\hline $\mathrm{Cu}$ & $\mathrm{Ta}$ & -1.541 & 0.243 & 0.04 & 0.443 & 1.358 & 0 & & $\mathrm{X}$ & $\mathrm{X}$ & $X$ & \\
\hline $\mathrm{Cu}$ & $\mathrm{Ti}$ & -1.869 & 0.042 & 0 & 0.561 & 2.373 & 0.109 & $\mathrm{X}$ & $\mathrm{X}$ & & $\mathrm{X}$ & $\mathrm{X}$ \\
\hline $\mathrm{Cu}$ & $\mathrm{Tl}$ & -1.456 & 0.051 & 0.109 & 0.102 & 0.042 & 0 & & & $\mathrm{X}$ & & \\
\hline $\mathrm{Cu}$ & $\mathrm{Y}$ & -2.135 & -0.03 & 2.841 & 3.11 & 4.543 & 0.07 & $\mathrm{X}$ & & $\mathrm{X}$ & $\mathrm{X}$ & \\
\hline $\mathrm{Cu}$ & $\mathrm{Zn}$ & -1.615 & 0.03 & 0.614 & 1.209 & 2.691 & 0.139 & $\mathrm{X}$ & $\mathrm{X}$ & & $\mathrm{X}$ & $\mathrm{X}$ \\
\hline $\mathrm{Fe}$ & $\mathrm{Ge}$ & -1.561 & 0.04 & 0.163 & 0.924 & 3.655 & 0 & $\mathrm{X}$ & & $\mathrm{X}$ & $\mathrm{X}$ & \\
\hline $\mathrm{Fe}$ & $\mathrm{K}$ & -1.845 & -0.014 & 0.804 & 1.58 & 3.184 & 0 & $\mathrm{X}$ & & $\mathrm{X}$ & $\mathrm{X}$ & \\
\hline $\mathrm{Fe}$ & $\mathrm{Mg}$ & -1.922 & 0.03 & 0.295 & 1.059 & 3.983 & 0.007 & $\mathrm{X}$ & & $X$ & $\mathrm{X}$ & \\
\hline $\mathrm{Fe}$ & $\mathrm{Mn}$ & -1.772 & 0.005 & 0.354 & 1.169 & 3.992 & 0.024 & $X$ & & $\mathrm{X}$ & $\mathrm{X}$ & \\
\hline $\mathrm{Fe}$ & $\mathrm{Na}$ & -1.844 & -0.026 & 0.707 & 1.479 & 2.98 & 0 & $\mathrm{X}$ & $\mathrm{X}$ & $\mathrm{X}$ & $\mathrm{X}$ & \\
\hline $\mathrm{Fe}$ & $\mathrm{Ni}$ & -1.61 & 0.033 & 0 & 0.036 & 2.622 & 0.062 & $\mathrm{X}$ & $\mathrm{X}$ & $\mathrm{X}$ & $\mathrm{X}$ & $\mathrm{X}$ \\
\hline $\mathrm{Fe}$ & $\mathrm{Pb}$ & -1.682 & 0.015 & 0.217 & 1.043 & 3.568 & 0.091 & X & & $\mathrm{X}$ & & \\
\hline $\mathrm{Fe}$ & $\mathrm{Pt}$ & -1.417 & 0.094 & 0.513 & 1.093 & 3.071 & 0.012 & & & $\mathrm{X}$ & $\mathrm{X}$ & \\
\hline $\mathrm{Fe}$ & $\mathrm{Rb}$ & -1.833 & -0.002 & 0.812 & 1.596 & 3.284 & 0.003 & $\mathrm{X}$ & & $\mathrm{X}$ & $\mathrm{X}$ & \\
\hline $\mathrm{Fe}$ & $\mathrm{Sn}$ & -1.625 & 0.014 & 0.166 & 0.966 & 2.892 & 0.669 & $X$ & $\mathrm{X}$ & & $\mathrm{X}$ & \\
\hline $\mathrm{Fe}$ & $\mathrm{Sr}$ & -2.12 & 0.004 & 0.227 & 0.998 & 3.837 & 0.035 & $X$ & & X & $\mathrm{X}$ & \\
\hline $\mathrm{Fe}$ & $\mathrm{Ti}$ & -1.847 & 0.087 & 0 & 0 & 1.926 & 0.191 & & $\mathrm{X}$ & & $X$ & X \\
\hline $\mathrm{Fe}$ & $\mathrm{Tl}$ & -1.634 & -0.013 & 0 & 0.441 & 1.828 & 0.922 & $\mathrm{X}$ & $\mathrm{X}$ & & & \\
\hline $\mathrm{Fe}$ & $\mathrm{Zn}$ & -1.671 & 0.069 & 0.312 & 1.045 & 3.833 & 0.009 & & & $\mathrm{X}$ & $\mathrm{X}$ & \\
\hline $\mathrm{Ga}$ & $\mathrm{Hg}$ & -1.584 & 0.071 & 3.01 & 3.388 & 3.942 & 0.061 & & & $\mathrm{X}$ & & \\
\hline $\mathrm{Ga}$ & $\mathrm{K}$ & -1.925 & 0.02 & 3.498 & 3.979 & 4.903 & 0 & $X$ & & $\mathrm{X}$ & $\mathrm{X}$ & \\
\hline $\mathrm{Ga}$ & $\mathrm{Na}$ & -1.917 & 0.015 & 2.848 & 3.31 & 4.212 & 0 & $\mathrm{X}$ & & $\mathrm{X}$ & $X$ & \\
\hline $\mathrm{Ga}$ & $\mathrm{Rb}$ & -1.914 & 0.031 & 3.66 & 4.15 & 5.07 & 0 & $\mathrm{X}$ & & $\mathrm{X}$ & $\mathrm{X}$ & \\
\hline $\mathrm{Ga}$ & $\mathrm{Tl}$ & -1.713 & 0.023 & 2.686 & 3.07 & 3.633 & 0.478 & $\mathrm{X}$ & & & & \\
\hline $\mathrm{Ge}$ & $\mathrm{Hg}$ & -1.453 & 0.055 & 0.907 & 1.062 & 1.678 & 0.615 & & $X$ & & & \\
\hline $\mathrm{Ge}$ & $\mathrm{Mg}$ & -1.912 & 0.013 & 3.274 & 3.65 & 4.212 & 0.251 & $\mathrm{X}$ & & & $\mathrm{X}$ & $\mathrm{X}$ \\
\hline $\mathrm{Ge}$ & $\mathrm{Mn}$ & -1.72 & 0.031 & 1.477 & 2.213 & 3.079 & 0.532 & $X$ & & & $X$ & \\
\hline $\mathrm{Ge}$ & $\mathrm{Pb}$ & -1.627 & 0.043 & 1.617 & 1.852 & 2.274 & 0 & $\mathrm{X}$ & $\mathrm{X}$ & $\mathrm{X}$ & & \\
\hline $\mathrm{Ge}$ & $\mathrm{Pd}$ & -1.416 & 0.071 & 0.092 & 0.092 & 0.193 & 0.166 & & & & $\mathrm{X}$ & \\
\hline $\mathrm{Ge}$ & $\mathrm{Pt}$ & -1.303 & 0.184 & 0.067 & 0.071 & 0 & 0 & & & $\mathrm{X}$ & $\mathrm{X}$ & \\
\hline $\mathrm{Ge}$ & $\mathrm{Sn}$ & -1.586 & 0.027 & 1.947 & 2.197 & 2.657 & 0 & $X$ & $X$ & $\mathrm{X}$ & $\mathrm{X}$ & \\
\hline $\mathrm{Ge}$ & $\mathrm{Sr}$ & -2.052 & 0.046 & 3.997 & 4.363 & 4.977 & 0 & $\mathrm{X}$ & & $\mathrm{X}$ & $\mathrm{X}$ & \\
\hline $\mathrm{Ge}$ & $\mathrm{Ti}$ & -1.757 & 0.163 & 0.148 & 0.864 & 2.293 & 0.105 & & $X$ & & $\mathrm{X}$ & \\
\hline $\mathrm{Ge}$ & $\mathrm{V}$ & -1.739 & 0.075 & 0.806 & 1.729 & 3.253 & 0.086 & & & $\mathrm{X}$ & $\mathrm{X}$ & \\
\hline $\mathrm{Ge}$ & $\mathrm{Zn}$ & -1.688 & 0.009 & 3.436 & 3.701 & 4.411 & 0.11 & $\mathrm{X}$ & & & $\mathrm{X}$ & $\mathrm{X}$ \\
\hline $\mathrm{Hg}$ & In & -1.585 & 0.074 & 0 & 0 & 0 & 0 & & & $\mathrm{X}$ & & \\
\hline $\mathrm{Hg}$ & $\mathrm{Ir}$ & -1.303 & 0.068 & 0 & 0.049 & 0.119 & 1.025 & & & & & $\mathrm{X}$ \\
\hline $\mathrm{Hg}$ & $\mathrm{Mg}$ & -1.804 & 0.055 & 1.709 & 2.14 & 2.976 & 0 & & $\mathrm{X}$ & $\mathrm{X}$ & & \\
\hline $\mathrm{Hg}$ & $\mathrm{Mn}$ & -1.651 & 0.033 & 0.092 & 0.374 & 1.47 & 0 & $X$ & $X$ & $\mathrm{X}$ & & \\
\hline $\mathrm{Hg}$ & Mo & -1.525 & 0.064 & 0 & 0.327 & 1.102 & 0.008 & & $\mathrm{X}$ & $\mathrm{X}$ & & $\mathrm{X}$ \\
\hline
\end{tabular}




\begin{tabular}{|c|c|c|c|c|c|c|c|c|c|c|c|c|}
\hline $\mathrm{Hg}$ & $\mathrm{Nb}$ & -1.687 & -0.02 & 0 & 0.474 & 1.56 & 0.001 & $\mathrm{X}$ & $\mathrm{X}$ & $\mathrm{X}$ & & \\
\hline $\mathrm{Hg}$ & $\mathrm{Ni}$ & -1.436 & 0.114 & 0.688 & 1.618 & 3.523 & 0.071 & & & $\mathrm{X}$ & & \\
\hline $\mathrm{Hg}$ & $\mathrm{Pb}$ & -1.559 & 0.045 & 1.668 & 1.928 & 2.627 & 0.582 & $\mathrm{X}$ & $\mathrm{X}$ & & & \\
\hline $\mathrm{Hg}$ & $\mathrm{Pd}$ & -1.401 & -0.003 & 1.279 & 1.504 & 2.538 & 0.18 & $\mathrm{X}$ & $\mathrm{X}$ & & & \\
\hline $\mathrm{Hg}$ & $\mathrm{Pt}$ & -1.344 & 0.066 & 0.943 & 1.202 & 1.254 & 0.08 & & $\mathrm{X}$ & $\mathrm{X}$ & & \\
\hline $\mathrm{Hg}$ & $\mathrm{Rh}$ & -1.383 & 0.047 & 0.062 & 0.087 & 0 & 0 & $\mathrm{X}$ & & $\mathrm{X}$ & & \\
\hline $\mathrm{Hg}$ & $\mathrm{Ru}$ & -1.326 & 0.118 & 0 & 0.19 & 0.548 & 0.063 & & & $\mathrm{X}$ & & \\
\hline $\mathrm{Hg}$ & $\mathrm{Sb}$ & -1.426 & 0.108 & 0.231 & 0.243 & 0.285 & 0 & & & $\mathrm{X}$ & & \\
\hline $\mathrm{Hg}$ & $\mathrm{Sc}$ & -1.976 & 0.141 & 0 & 0 & 0.577 & 2.294 & & & & & \\
\hline $\mathrm{Hg}$ & $\mathrm{Sn}$ & -1.511 & 0.095 & 0.849 & 1.011 & 1.588 & 0.534 & & $\mathrm{X}$ & & & \\
\hline $\mathrm{Hg}$ & $\mathrm{Sr}$ & -1.987 & 0.059 & 2.867 & 3.263 & 4.2 & 0.318 & & & & & \\
\hline $\mathrm{Hg}$ & $\mathrm{Ta}$ & -1.72 & 0.064 & 0 & 0.434 & 1.429 & 0.02 & & $\mathrm{X}$ & $\mathrm{X}$ & & $\mathrm{X}$ \\
\hline $\mathrm{Hg}$ & $\mathrm{Ti}$ & -1.833 & 0.077 & 1.263 & 1.607 & 2.04 & 0.026 & & $\mathrm{X}$ & $\mathrm{X}$ & & \\
\hline $\mathrm{Hg}$ & $\mathrm{Tl}$ & -1.456 & 0.043 & 0.553 & 0.673 & 1.165 & 0.495 & $\mathrm{X}$ & $\mathrm{X}$ & & & \\
\hline $\mathrm{Hg}$ & $\mathrm{V}$ & -1.635 & 0.15 & 0.078 & 0.7 & 1.302 & 0.038 & & $\mathrm{X}$ & X & & \\
\hline $\mathrm{Hg}$ & $\mathrm{Y}$ & -2.055 & 0.055 & 0 & 0.282 & 0.877 & 2.682 & & & & & \\
\hline $\mathrm{Hg}$ & $\mathrm{Zn}$ & -1.555 & 0.078 & 1.56 & 1.859 & 2.721 & 0.073 & & $\mathrm{X}$ & $\mathrm{X}$ & & \\
\hline In & $\mathrm{K}$ & -1.973 & -0.014 & 3.72 & 4.233 & 5.11 & 0 & $\mathrm{X}$ & & $\mathrm{X}$ & $\mathrm{X}$ & \\
\hline In & $\mathrm{Li}$ & -1.949 & -0.003 & 2.902 & 3.397 & 4.251 & 0 & $\mathrm{X}$ & & $\mathrm{X}$ & $\mathrm{X}$ & \\
\hline In & $\mathrm{Na}$ & -1.974 & -0.028 & 3.088 & 3.597 & 4.453 & 0 & $\mathrm{X}$ & & $\mathrm{X}$ & $\mathrm{X}$ & \\
\hline In & $\mathrm{Rb}$ & -1.967 & -0.007 & 3.824 & 4.34 & 5.216 & 0.035 & $\mathrm{X}$ & & $\mathrm{X}$ & $\mathrm{X}$ & \\
\hline In & $\mathrm{Tl}$ & -1.758 & -0.009 & 2.816 & 3.221 & 3.795 & 0.399 & $\mathrm{X}$ & & & & \\
\hline $\mathrm{Ir}$ & $\mathrm{K}$ & -1.662 & -0.02 & 1.904 & 2.423 & 3.562 & 0 & $\mathrm{X}$ & & $\mathrm{X}$ & $\mathrm{X}$ & \\
\hline $\mathrm{Ir}$ & $\mathrm{Li}$ & -1.641 & -0.013 & 1.522 & 1.887 & 2.974 & 0 & $\mathrm{X}$ & $\mathrm{X}$ & $\mathrm{X}$ & $\mathrm{X}$ & $\mathrm{X}$ \\
\hline $\mathrm{Ir}$ & $\mathrm{Na}$ & -1.643 & -0.015 & 1.466 & 1.981 & 3.101 & 0 & $X$ & & X & $\mathrm{X}$ & \\
\hline $\mathrm{Ir}$ & $\mathrm{Rb}$ & -1.654 & -0.012 & 2.002 & 2.528 & 3.664 & 0 & $\mathrm{X}$ & & $\mathrm{X}$ & $\mathrm{X}$ & \\
\hline $\mathrm{Ir}$ & $\mathrm{Tl}$ & -1.456 & -0.025 & 1.723 & 2.194 & 3.286 & 0.059 & $\mathrm{X}$ & & $\mathrm{X}$ & & \\
\hline $\mathrm{K}$ & $\mathrm{Mn}$ & -1.939 & 0.021 & 0.396 & 0.73 & 1.862 & 0.005 & $\mathrm{X}$ & $\mathrm{X}$ & $\mathrm{X}$ & $\mathrm{X}$ & \\
\hline $\mathrm{K}$ & Mo & -1.886 & 0.004 & 1.603 & 2.328 & 3.646 & 0.224 & $\mathrm{X}$ & & & $\mathrm{X}$ & \\
\hline $\mathrm{K}$ & $\mathrm{Nb}$ & -1.98 & -0.026 & 0 & 0.341 & 1.71 & 0.006 & $\mathrm{X}$ & $\mathrm{X}$ & X & $\mathrm{X}$ & \\
\hline $\mathrm{K}$ & $\mathrm{Ni}$ & -1.703 & 0.014 & 0.195 & 0.326 & 1.317 & 0.07 & $\mathrm{X}$ & $\mathrm{X}$ & X & $\mathrm{X}$ & \\
\hline $\mathrm{K}$ & $\mathrm{Pd}$ & -1.635 & 0.016 & 0.375 & 0.678 & 1.772 & 0.046 & $\mathrm{X}$ & $\mathrm{X}$ & $\mathrm{X}$ & $\mathrm{X}$ & \\
\hline $\mathrm{K}$ & $\mathrm{Rh}$ & -1.758 & -0.054 & 1.644 & 2.273 & 3.449 & 0 & $\mathrm{X}$ & & $\mathrm{X}$ & $\mathrm{X}$ & \\
\hline $\mathrm{K}$ & $\mathrm{Ru}$ & -1.71 & 0.033 & 0 & 0.56 & 2.067 & 0.011 & $\mathrm{X}$ & $\mathrm{X}$ & $\mathrm{X}$ & $\mathrm{X}$ & \\
\hline $\mathrm{K}$ & $\mathrm{Sb}$ & -1.798 & 0.035 & 3.543 & 3.811 & 4.402 & 0.023 & $\mathrm{X}$ & & $\mathrm{X}$ & $\mathrm{X}$ & \\
\hline $\mathrm{K}$ & $\mathrm{Sc}$ & -2.403 & 0.014 & 4.024 & 4.486 & 5.571 & 0 & $\mathrm{X}$ & & $\mathrm{X}$ & $\mathrm{X}$ & \\
\hline $\mathrm{K}$ & $\mathrm{Ti}$ & -2.17 & 0.021 & 0.043 & 0.668 & 2.632 & 0.01 & $\mathrm{X}$ & $\mathrm{X}$ & X & $\mathrm{X}$ & \\
\hline $\mathrm{K}$ & $\mathrm{Tl}$ & -1.73 & 0.036 & 2.327 & 2.594 & 3.476 & 0.029 & $\mathrm{X}$ & & $\mathrm{X}$ & & \\
\hline $\mathrm{K}$ & $\mathrm{V}$ & -2.059 & 0.026 & 0.134 & 0.976 & 2.987 & 0 & $\mathrm{X}$ & $X$ & $\mathrm{X}$ & $\mathrm{X}$ & \\
\hline $\mathrm{K}$ & $\mathrm{Y}$ & -2.487 & -0.096 & 5.336 & 5.889 & 6.813 & 0.028 & $\mathrm{X}$ & & $\mathrm{X}$ & $\mathrm{X}$ & \\
\hline $\mathrm{Li}$ & Mo & -1.891 & -0.014 & 1.565 & 2.264 & 3.389 & 0 & $\mathrm{X}$ & & $\mathrm{X}$ & $\mathrm{X}$ & X \\
\hline $\mathrm{Li}$ & $\mathrm{Nb}$ & -1.958 & -0.009 & 0 & 0.292 & 1.462 & 0.002 & $\mathrm{X}$ & $X$ & $\mathrm{X}$ & $\mathrm{X}$ & \\
\hline
\end{tabular}




\begin{tabular}{|c|c|c|c|c|c|c|c|c|c|c|c|c|}
\hline $\mathrm{Li}$ & $\mathrm{Pd}$ & -1.608 & -0.037 & 0.171 & 0.394 & 1.328 & 0.69 & X & $\mathrm{X}$ & & $\mathrm{X}$ & \\
\hline $\mathrm{Li}$ & $\mathrm{Ru}$ & -1.702 & 0.028 & 0 & 0.44 & 1.909 & 0 & X & $\mathrm{X}$ & $\mathrm{X}$ & $\mathrm{X}$ & $\mathrm{X}$ \\
\hline $\mathrm{Li}$ & $\mathrm{Sb}$ & -1.781 & 0.039 & 2.984 & 3.267 & 3.801 & 0.125 & $X$ & & & X & \\
\hline $\mathrm{Li}$ & $\mathrm{Sc}$ & -2.383 & 0.02 & 3.705 & 4.146 & 5.211 & 0 & $X$ & & $X$ & $\mathrm{X}$ & \\
\hline $\mathrm{Li}$ & $\mathrm{Ta}$ & -1.859 & 0.13 & 0 & 0 & 1.146 & 0 & & $X$ & $X$ & $X$ & \\
\hline $\mathrm{Li}$ & $\mathrm{Tl}$ & -1.711 & 0.03 & 1.445 & 1.698 & 2.583 & 0 & $X$ & $\mathrm{X}$ & $X$ & & \\
\hline $\mathrm{Li}$ & $\mathrm{Y}$ & -2.47 & -0.071 & 4.927 & 5.481 & 6.372 & 0 & $X$ & & $X$ & $\mathrm{X}$ & \\
\hline $\mathrm{Mg}$ & $\mathrm{Mn}$ & -2.088 & 0.014 & 2.425 & 3.59 & 4.817 & 0 & $\mathrm{X}$ & & $\mathrm{X}$ & $\mathrm{X}$ & \\
\hline $\mathrm{Mg}$ & $\mathrm{Pb}$ & -2.001 & 0.021 & 3.921 & 4.367 & 4.908 & 0.224 & $\mathrm{X}$ & & & & \\
\hline $\mathrm{Mg}$ & $\mathrm{Pd}$ & -1.77 & 0.046 & 1.86 & 2.235 & 3.461 & 0.041 & X & & $X$ & $\mathrm{X}$ & \\
\hline $\mathrm{Mg}$ & $\mathrm{Pt}$ & -1.734 & 0.094 & 2.274 & 2.774 & 3.853 & 0 & & & $\mathrm{X}$ & $X$ & \\
\hline $\mathrm{Mg}$ & $\mathrm{Sn}$ & -1.94 & 0.024 & 3.191 & 3.599 & 4.074 & 0.242 & X & & & $X$ & \\
\hline $\mathrm{Mg}$ & $\mathrm{Sr}$ & -2.448 & 0.001 & 5.268 & 6.009 & 6.633 & 0 & X & & $\mathrm{X}$ & $\mathrm{X}$ & \\
\hline $\mathrm{Mg}$ & $\mathrm{Ti}$ & -2.132 & 0.024 & 0.124 & 0.938 & 2.714 & 0 & X & $X$ & $\mathrm{X}$ & X & \\
\hline $\mathrm{Mg}$ & V & -2.11 & 0.048 & 0.859 & 2.014 & 3.646 & 0 & X & & $\mathrm{X}$ & $\mathrm{X}$ & \\
\hline $\mathrm{Mg}$ & $\mathrm{Zn}$ & -2.016 & 0.048 & 4.447 & 4.904 & 5.783 & 0.011 & X & & $\mathrm{X}$ & X & X \\
\hline $\mathrm{Mn}$ & $\mathrm{Na}$ & -1.937 & -0.058 & 0.272 & 0.575 & 1.639 & 0.164 & $X$ & $\mathrm{X}$ & & $X$ & \\
\hline $\mathrm{Mn}$ & $\mathrm{Ni}$ & -1.669 & 0.124 & 0 & 0.797 & 2.512 & 0.302 & & $X$ & & $X$ & \\
\hline $\mathrm{Mn}$ & $\mathrm{Pb}$ & -1.84 & 0.013 & 1.705 & 2.636 & 3.629 & 0.388 & X & & & & \\
\hline $\mathrm{Mn}$ & $\mathrm{Pt}$ & -1.556 & 0.097 & 1.077 & 1.792 & 3.132 & 0.257 & & & & $\mathrm{X}$ & \\
\hline $\mathrm{Mn}$ & $\mathrm{Rb}$ & -1.93 & 0.048 & 0.498 & 0.829 & 1.946 & 0.048 & X & $X$ & $X$ & $\mathrm{X}$ & \\
\hline $\mathrm{Mn}$ & $\mathrm{Sn}$ & -1.782 & 0.013 & 1.349 & 2.214 & 3.021 & 0.414 & X & & & $X$ & \\
\hline $\mathrm{Mn}$ & $\mathrm{Sr}$ & -2.278 & 0.027 & 2.448 & 3.577 & 5.532 & 0.419 & $\mathrm{X}$ & & & $\mathrm{X}$ & \\
\hline $\mathrm{Mn}$ & $\mathrm{Ti}$ & -1.972 & 0.037 & 0.103 & 0.819 & 2.541 & 0.098 & $\mathrm{X}$ & $\mathrm{X}$ & $\mathrm{X}$ & $\mathrm{X}$ & \\
\hline $\mathrm{Mn}$ & $\mathrm{Tl}$ & -1.746 & 0.005 & 0.1 & 0.091 & 0 & 0 & $\mathrm{X}$ & & $\mathrm{X}$ & & \\
\hline $\mathrm{Mn}$ & V & -1.95 & 0.034 & 0.622 & 1.836 & 3.501 & 0 & X & & $\mathrm{X}$ & $\mathrm{X}$ & \\
\hline $\mathrm{Mn}$ & $\mathrm{Zn}$ & -1.817 & 0.075 & 2.489 & 3.581 & 5.064 & 0.036 & & & $X$ & $\mathrm{X}$ & \\
\hline Mo & $\mathrm{Na}$ & -1.883 & -0.006 & 1.538 & 2.254 & 3.475 & 0 & X & & $\mathrm{X}$ & X & \\
\hline Mo & $\mathrm{Rb}$ & -1.877 & 0.014 & 1.655 & 2.375 & 3.7 & 0.252 & $X$ & & & $\mathrm{X}$ & \\
\hline Mo & $\mathrm{Tl}$ & -1.669 & 0.011 & 1.629 & 2.376 & 3.521 & 0.064 & X & & X & & \\
\hline $\mathrm{Na}$ & $\mathrm{Nb}$ & -1.983 & -0.056 & 0 & 0.33 & 1.486 & 0 & X & X & X & X & \\
\hline $\mathrm{Na}$ & $\mathrm{Ni}$ & -1.693 & 0.011 & 0.222 & 0.317 & 1.1 & 0 & X & X & X & X & \\
\hline $\mathrm{Na}$ & $\mathrm{Pd}$ & -1.63 & -0.048 & 0.057 & 0.455 & 1.358 & 0.043 & X & X & X & X & \\
\hline $\mathrm{Na}$ & $\mathrm{Rh}$ & -1.742 & -0.062 & 1.256 & 1.883 & 3.026 & 0 & X & & $\mathrm{X}$ & X & \\
\hline $\mathrm{Na}$ & $\mathrm{Ru}$ & -1.7 & 0.03 & 0 & 0.458 & 1.962 & 0 & X & X & $\mathrm{X}$ & X & \\
\hline $\mathrm{Na}$ & $\mathrm{Sb}$ & -1.798 & 0.023 & 3.152 & 3.436 & 3.985 & 0.141 & X & & & $\mathrm{X}$ & \\
\hline $\mathrm{Na}$ & $\mathrm{Sc}$ & -2.407 & -0.004 & 3.83 & 4.28 & 5.36 & 0 & $\mathrm{X}$ & & $X$ & $\mathrm{X}$ & \\
\hline $\mathrm{Na}$ & $\mathrm{Ti}$ & -2.172 & 0.006 & 0.059 & 0.678 & 2.694 & 0.044 & X & $\mathrm{X}$ & $\mathrm{X}$ & X & \\
\hline $\mathrm{Na}$ & $\mathrm{Tl}$ & -1.73 & 0.022 & 1.687 & 1.957 & 2.853 & 0 & X & $\mathrm{X}$ & $\mathrm{X}$ & & \\
\hline $\mathrm{Na}$ & V & -2.057 & 0.015 & 0.157 & 1.049 & 2.969 & 0 & X & $X$ & X & X & \\
\hline $\mathrm{Na}$ & $\mathrm{Y}$ & -2.489 & -0.111 & 5.078 & 5.633 & 6.53 & 0 & X & & $\mathrm{X}$ & $\mathrm{X}$ & \\
\hline $\mathrm{Nb}$ & $\mathrm{Rb}$ & -1.972 & 0.005 & 0.074 & 0.548 & 1.792 & 0.029 & X & X & $\mathrm{X}$ & $\mathrm{X}$ & \\
\hline
\end{tabular}




\begin{tabular}{|c|c|c|c|c|c|c|c|c|c|c|c|c|}
\hline $\mathrm{Ni}$ & $\mathrm{Pb}$ & -1.678 & 0.034 & 0.451 & 1.508 & 2.87 & 1.098 & $X$ & $X$ & & & \\
\hline $\mathrm{Ni}$ & $\mathrm{Pt}$ & -1.369 & 0.15 & 0.509 & 1.406 & 2.903 & 0.079 & & $\mathrm{X}$ & $\mathrm{X}$ & $\mathrm{X}$ & \\
\hline $\mathrm{Ni}$ & $\mathrm{Rb}$ & -1.694 & 0.023 & 0.242 & 0.386 & 1.397 & 0.072 & $X$ & $X$ & $X$ & X & \\
\hline $\mathrm{Ni}$ & $\mathrm{Sn}$ & -1.623 & 0.032 & 0 & 0.925 & 2.132 & 1.059 & $X$ & $X$ & & $\mathrm{X}$ & \\
\hline $\mathrm{Ni}$ & $\mathrm{Sr}$ & -2.029 & 0.111 & 0.765 & 1.682 & 3.724 & 0.117 & & & & $X$ & \\
\hline $\mathrm{Ni}$ & $\mathrm{Ti}$ & -1.774 & 0.167 & 0 & 0.103 & 1.641 & 0.074 & & $X$ & $X$ & $\mathrm{X}$ & \\
\hline $\mathrm{Ni}$ & $\mathrm{Tl}$ & -1.555 & 0.052 & 0 & 0 & 0 & 0 & & & $X$ & & \\
\hline $\mathrm{Ni}$ & $\mathrm{V}$ & -1.798 & 0.051 & 0.311 & 0.311 & 2.029 & 0 & & $\mathrm{X}$ & $\mathrm{X}$ & $\mathrm{X}$ & $\mathrm{X}$ \\
\hline $\mathrm{Pb}$ & $\mathrm{Pd}$ & -1.529 & 0.034 & 0 & 0.131 & 0.939 & 1.839 & $\mathrm{X}$ & & & & \\
\hline $\mathrm{Pb}$ & $\mathrm{Pt}$ & -1.463 & 0.111 & 1.598 & 1.972 & 2.892 & 0.193 & & $X$ & & & \\
\hline $\mathrm{Pb}$ & $\mathrm{Sn}$ & -1.686 & 0.023 & 1.692 & 1.945 & 2.346 & 0 & $X$ & $\mathrm{X}$ & $\mathrm{X}$ & & \\
\hline $\mathrm{Pb}$ & $\mathrm{Sr}$ & -2.18 & 0.014 & 4.552 & 4.958 & 5.581 & 0.001 & X & & $\mathrm{X}$ & & \\
\hline $\mathrm{Pb}$ & $\mathrm{Ti}$ & -1.879 & 0.089 & 0.169 & 0.885 & 2.36 & 0.085 & & $X$ & $\mathrm{X}$ & & \\
\hline $\mathrm{Pb}$ & V & -1.866 & 0.038 & 0.974 & 1.916 & 3.4 & 0.081 & $\mathrm{X}$ & & $\mathrm{X}$ & & \\
\hline $\mathrm{Pb}$ & $\mathrm{Zn}$ & -1.739 & 0.079 & 2.764 & 3.18 & 3.814 & 0.563 & & & & & \\
\hline $\mathrm{Pd}$ & $\mathrm{Pt}$ & -1.283 & 0.097 & 0 & 0.067 & 0.281 & 0.392 & & & & X & \\
\hline $\mathrm{Pd}$ & $\mathrm{Rb}$ & -1.629 & 0.037 & 0.397 & 0.773 & 1.868 & 0.083 & $X$ & $\mathrm{X}$ & $\mathrm{X}$ & $X$ & \\
\hline $\mathrm{Pd}$ & $\mathrm{Sn}$ & -1.491 & 0.115 & 0.147 & 0.143 & 0.136 & 0.168 & & & & $X$ & \\
\hline $\mathrm{Pd}$ & $\mathrm{Sr}$ & -1.962 & 0.047 & 1.952 & 2.328 & 3.575 & 0.034 & $\mathrm{X}$ & & $X$ & $X$ & \\
\hline $\mathrm{Pd}$ & $\mathrm{Tl}$ & -1.466 & -0.003 & 0.125 & 0.125 & 0.092 & 0.194 & $\mathrm{X}$ & & & & \\
\hline $\mathrm{Pd}$ & $\mathrm{Y}$ & -2.06 & 0.02 & 0.532 & 1.127 & 2.801 & 0.02 & $\mathrm{X}$ & $X$ & $X$ & $\mathrm{X}$ & \\
\hline $\mathrm{Pd}$ & $\mathrm{Zn}$ & -1.541 & 0.076 & 1.37 & 1.646 & 2.932 & 0.087 & & $\mathrm{X}$ & $\mathrm{X}$ & $X$ & \\
\hline $\mathrm{Pt}$ & $\mathrm{Sn}$ & -1.368 & 0.239 & 0.056 & 0.075 & 0.114 & 0.115 & & & & $\mathrm{X}$ & \\
\hline $\mathrm{Pt}$ & $\mathrm{Sr}$ & -1.929 & 0.072 & 2.414 & 2.877 & 4.007 & 0.011 & & & $\mathrm{X}$ & $\mathrm{X}$ & \\
\hline $\mathrm{Pt}$ & $\mathrm{Ti}$ & -1.63 & 0.28 & 0.417 & 0.364 & 1.024 & 0.017 & & $\mathrm{X}$ & $\mathrm{X}$ & $\mathrm{X}$ & $\mathrm{X}$ \\
\hline $\mathrm{Pt}$ & V & -1.6 & 0.165 & 0.424 & 0.987 & 2.265 & 0.139 & & $\mathrm{X}$ & & $\mathrm{X}$ & \\
\hline $\mathrm{Pt}$ & $\mathrm{Zn}$ & -1.496 & 0.104 & 2.356 & 2.816 & 3.952 & 0.013 & & & $X$ & $\mathrm{X}$ & \\
\hline $\mathrm{Rb}$ & $\mathrm{Rh}$ & -1.75 & -0.056 & 1.728 & 2.363 & 3.542 & 0 & $\mathrm{X}$ & & $\mathrm{X}$ & $\mathrm{X}$ & \\
\hline $\mathrm{Rb}$ & $\mathrm{Sb}$ & -1.795 & 0.039 & 3.55 & 3.817 & 4.409 & 0.027 & $\mathrm{X}$ & & $X$ & $\mathrm{X}$ & \\
\hline $\mathrm{Rb}$ & $\mathrm{Sc}$ & -2.393 & 0.024 & 4.069 & 4.532 & 5.617 & 0 & $\mathrm{X}$ & & $\mathrm{X}$ & $X$ & \\
\hline $\mathrm{Rb}$ & $\mathrm{Ta}$ & -1.917 & 0.086 & 0 & 0.368 & 1.321 & 0 & & $X$ & $X$ & $\mathrm{X}$ & $\mathrm{X}$ \\
\hline $\mathrm{Rb}$ & $\mathrm{Ti}$ & -2.159 & 0.033 & 0.09 & 0.718 & 2.695 & 0 & $\mathrm{X}$ & $X$ & $\mathrm{X}$ & X & \\
\hline $\mathrm{Rb}$ & $\mathrm{Tl}$ & -1.725 & 0.041 & 2.424 & 2.695 & 3.598 & 0.053 & $X$ & & $X$ & & \\
\hline $\mathrm{Rb}$ & $\mathrm{V}$ & -2.048 & 0.037 & 0.217 & 1.115 & 3.069 & 0.015 & $\mathrm{X}$ & & $\mathrm{X}$ & $\mathrm{X}$ & \\
\hline $\mathrm{Rb}$ & $\mathrm{Y}$ & -2.482 & -0.064 & 5.351 & 5.906 & 6.767 & 0.043 & $\mathrm{X}$ & & $\mathrm{X}$ & $X$ & \\
\hline $\mathrm{Rh}$ & $\mathrm{Tl}$ & -1.558 & -0.074 & 1.535 & 2.131 & 3.128 & 0.077 & $\mathrm{X}$ & & $\mathrm{X}$ & & \\
\hline $\mathrm{Sb}$ & $\mathrm{Tl}$ & -1.59 & 0.033 & 1.862 & 2.079 & 2.51 & 0 & $\mathrm{X}$ & $X$ & $X$ & & \\
\hline $\mathrm{Sc}$ & $\mathrm{Tl}$ & -2.181 & 0.025 & 3.477 & 3.903 & 4.739 & 0 & $\mathrm{X}$ & & $\mathrm{X}$ & & \\
\hline $\mathrm{Sn}$ & $\mathrm{Sr}$ & -2.116 & 0.02 & 3.822 & 4.192 & 4.748 & 0.021 & $\mathrm{X}$ & & $\mathrm{X}$ & $\mathrm{X}$ & \\
\hline $\mathrm{Sn}$ & $\mathrm{Ti}$ & -1.82 & 0.12 & 0.198 & 0.93 & 2.356 & 0.077 & & $\mathrm{X}$ & $\mathrm{X}$ & $\mathrm{X}$ & \\
\hline $\mathrm{Sn}$ & $\mathrm{V}$ & -1.807 & 0.039 & 0.917 & 1.893 & 3.36 & 0.082 & $\mathrm{X}$ & & $X$ & $X$ & \\
\hline $\mathrm{Sn}$ & $\mathrm{Zn}$ & -1.682 & 0.074 & 2.119 & 2.484 & 3.034 & 0.575 & & & & $\mathrm{X}$ & \\
\hline
\end{tabular}




\begin{tabular}{|c|c|c|c|c|c|c|c|c|c|c|c|c|}
\hline $\mathrm{Sr}$ & $\mathrm{Ti}$ & -2.324 & 0.004 & 0.234 & 0.983 & 2.718 & 0.065 & $\mathrm{X}$ & $X$ & $X$ & $\mathrm{X}$ & \\
\hline $\mathrm{Sr}$ & $\mathrm{V}$ & -2.312 & 0.018 & 1.402 & 2.574 & 4.268 & 0 & $X$ & & $X$ & $\mathrm{X}$ & \\
\hline $\mathrm{Sr}$ & $\mathrm{Zn}$ & -2.176 & 0.094 & 4.266 & 4.942 & 5.795 & 0 & & & $X$ & $\mathrm{X}$ & \\
\hline $\mathrm{Ti}$ & $\mathrm{Tl}$ & -1.952 & 0.03 & 0.136 & 0.753 & 2.74 & 0.003 & $\mathrm{X}$ & $X$ & $X$ & & \\
\hline $\mathrm{Ti}$ & $\mathrm{V}$ & -1.992 & 0.046 & 0.072 & 0.881 & 2.649 & 0.174 & $X$ & $\mathrm{X}$ & & $\mathrm{X}$ & \\
\hline $\mathrm{Ti}$ & $\mathrm{Zn}$ & -1.856 & 0.125 & 0.15 & 0.912 & 2.203 & 0.02 & & $\mathrm{X}$ & $\mathrm{X}$ & $\mathrm{X}$ & \\
\hline $\mathrm{Tl}$ & $\mathrm{V}$ & -1.843 & 0.032 & 0.162 & 1.09 & 2.899 & 0.004 & $\mathrm{X}$ & $\mathrm{X}$ & $X$ & & \\
\hline $\mathrm{Tl}$ & $\mathrm{Y}$ & -2.303 & -0.122 & 4.533 & 4.82 & 5.476 & 0.004 & $\mathrm{X}$ & & $\mathrm{X}$ & & $\mathrm{X}$ \\
\hline $\mathrm{V}$ & $\mathrm{Zn}$ & -1.832 & 0.101 & 0.197 & 1.232 & 2.861 & 0 & & $X$ & $\mathrm{X}$ & $\mathrm{X}$ & \\
\hline
\end{tabular}

